\title{
10. INTERSTITIAL WATER STUDIES, LEG 40: SHIPBOARD STUDIES
}

\author{
Victor Sotelo and Joris M. Gieskes, Scripps Institution of Oceanography, La Jolla, California
}

\begin{abstract}
Data on the distribution with depth of chlorinity and on dissolved calcium and magnesium suggest the influence of evaporite beds on the chemistry of the interstitial waters in sites in the Angola Basin (Sites 364 and 365), as well as in the Cape Basin (Sites 361 and 362), although in the latter the influence is small. Presumably, evaporite beds on the continental shelf and/or underlying salt layers are responsible for these observations.
\end{abstract}

\section{INTRODUCTION}

Here we present very briefly the results of the chemical analyses of the interstitial waters carried out by one of us (V.S.) onboard Glomar Challenger. The methods used have been described by Gieskes (1974), and the shipboard calcium and magnesium data have been recalculated using the formulae given by Gieskes and Lawrence (1976). Chlorinity data are precise to within $1 \%$, but from site to site differences of up to $3 \%$ are due to calibration errors.

No further shore laboratory studies have been made on these samples, but it should be emphasized that samples have been stored in both plastic vials and glass ampuls for possible further studies of chemical components. These samples are at the La Jolla, California, repository of the Deep Sea Drilling Project.

\section{RESULTS}

The results are presented in Table 1, and, for convenience, they are also presented in Figures 1 through 5.

\section{DISCUSSION}

\section{Site 360, Cape Basin}

Gradients in calcium and magnesium are very small, implying little reaction involving calcium and magnesium exchange with the pore fluids.

\section{Site 361, Cape Basin}

This site is of some interest because of the increased chlorinities and salinities at depth. The scatter in the chlorinity data seems to be about $2 \%$, probably due to dilution effects from distilled water in the squeezers. The low chlorinity at 918 meters is especially suspect because of the very small sample size and easy dilution with remnant water from the squeezer. On the other hand, the maximum in the Unit 7 (Aptian) sandstones at 1050 meters appears real. It is possible that advection of more saline water, also having higher magnesium and calcium concentrations, occurs in these sandstones. Perhaps this water originates in buried salt deposits on the shelf or on land.

\section{Site 362, Walvis Ridge}

This site is again characterized by a very small increase in chlorinity in the concentration-depth profile. Salinities drop to values of $33^{\circ} \%$ in the upper 400 meters as a result of large sulfate depletions (Gieskes, 1974), also reflected in the fairly high alkalinity values and in the initial decrease in dissolved calcium (as a result of calcium carbonate precipitation). The very definite increase in salinity and chlorinity with depth is suggestive of effects of deeper seated salt deposits at or near this site. A drill site just south of the Walvis Ridge would have been most instructive with regard to this.

\section{Site 363, Frio Ridge Portion of Walvis Ridge}

This site indicates very little change in chlorinity with depth, although perhaps a small increase is noticeable. Calcium and magnesium concentration profiles show a smooth linear concentration change with depth. Perhaps alteration processes in the underlying (igneous) basement high are responsible for these changes in calcium and magnesium.

\section{Site 364, Angola Basin Marginal Plateau}

Increases in chlorinity and salinity are smooth. At 900 meters, the chlorinity has reached a value of about $25 \%$ of the saturation chloride concentration for typical halite (Manheim et al., 1974). Thus, it appears that the salt layers are still at considerable depth below this site.

\section{Site 365, Canyon in Angola Basin Marginal Plateau}

In this site, chlorinites reached values about $40 \%$ of the halite saturation value. The depth profile does not suggest simple diffusion of chloride from underlying salt horizons. Also, below 400 meters, magnesium concentrations decrease considerably. Perhaps here the increased chloride concentrations are due to the horizontal advection of brines from salt deposits situated more toward the side of the submarine canyon in which these sediments were cored or toward the African coast. Similar observations have been made at Sites 369 and 370 of Leg 41 (Couture et al., 1977). The chemistry of Sample 1-2, 144-150 cm suggests that this 
TABLE 1

Interstitial Water Data, Leg 40

\begin{tabular}{|c|c|c|c|c|c|c|c|}
\hline Sample & $\begin{array}{c}\text { Depth } \\
(\mathrm{m})\end{array}$ & $p \mathrm{H}$ & $\begin{array}{c}\text { Alkalinity } \\
(\mathrm{meq} / \mathrm{l})\end{array}$ & $\begin{array}{c}\text { Chlorinity } \\
(\% \circ)\end{array}$ & $\begin{array}{c}\text { Salinity } \\
(\% \circ)\end{array}$ & $\begin{array}{c}\text { Calcium } \\
(\mathrm{mM})\end{array}$ & $\begin{array}{l}\text { Magnesium } \\
(\mathrm{mM})\end{array}$ \\
\hline \multicolumn{8}{|l|}{ Site 360} \\
\hline $\begin{array}{l}1-4,140-150 \\
5-4,144-150 \\
14-4,144-150 \\
20-3,144-150 \\
27-3,144-150\end{array}$ & $\begin{array}{r}87.5 \\
125.5 \\
211.0 \\
287.0 \\
420.0\end{array}$ & $\begin{array}{l}7.27 \\
7.39 \\
7.51 \\
7.66 \\
7.88\end{array}$ & $\begin{array}{l}5.57 \\
4.32 \\
2.07 \\
1.80 \\
0.25\end{array}$ & $\begin{array}{l}- \\
- \\
- \\
-\end{array}$ & $\begin{array}{l}34.6 \\
33.6 \\
33.8 \\
33.8 \\
34.1\end{array}$ & $\begin{array}{l}11.5 \\
11.3 \\
12.5 \\
13.3 \\
17.3\end{array}$ & $\begin{array}{c}48.5 \\
41.4 \\
40.0 \\
37.4 \\
(11.6 ?)\end{array}$ \\
\hline \multicolumn{8}{|l|}{ Site 361} \\
\hline $\begin{array}{l}1-2,144-150 \\
5-5,140-150 \\
8-5,140-150 \\
12-4,140-150 \\
14-1,140-150 \\
20-1,140-150 \\
22-3,140-150 \\
24-3,140-150 \\
26-4,140-150 \\
27-3,123-135 \\
31-4,65-75 \\
36-2,30-40 \\
40-3,83-93 \\
43-4,108-118 \\
46-2,100-109 \\
48-1,60-70\end{array}$ & $\begin{array}{r}35.0 \\
182.0 \\
258.0 \\
305.5 \\
372.0 \\
615.5 \\
723.5 \\
818.5 \\
912.0 \\
961.0 \\
1056 \\
1102 \\
1152 \\
1209 \\
1267 \\
1295\end{array}$ & $\begin{array}{l}7.52 \\
8.11 \\
7.40 \\
7.52 \\
7.88 \\
\\
8.01\end{array}$ & $\begin{array}{l}2.10 \\
0.58 \\
1.31 \\
1.38 \\
1.12 \\
0.82 \\
0.98 \\
1.56 \\
- \\
- \\
1.92 \\
1.24 \\
0.86 \\
0.92 \\
- \\
2.25\end{array}$ & $\begin{array}{l}19.2 \\
19.0 \\
19.4 \\
18.9 \\
19.4 \\
18.6 \\
19.4 \\
18.7 \\
(18.1) \\
\\
21.4 \\
20.4 \\
19.4 \\
19.6 \\
19.9 \\
20.4\end{array}$ & $\begin{array}{l}35.2 \\
34.1 \\
34.6 \\
33.6 \\
34.6 \\
31.4 \\
33.0 \\
31.4 \\
30.8 \\
\\
38.5 \\
34.9 \\
33.6 \\
35.5 \\
34.6 \\
-\end{array}$ & $\begin{array}{l}14.5 \\
23.3 \\
24.8 \\
28.5 \\
29.5 \\
30.7 \\
32.9 \\
35.3 \\
- \\
- \\
55.8 \\
46.8 \\
47.2 \\
58.6 \\
62.8 \\
60.9\end{array}$ & $\begin{array}{l}48.2 \\
35.0 \\
29.6 \\
26.0 \\
26.7 \\
16.2 \\
15.1 \\
11.3\end{array}$ \\
\hline \multicolumn{8}{|l|}{ Hole 362} \\
\hline $\begin{array}{l}1-4,144-150 \\
5-5,144-150 \\
10-5,144-150 \\
15-4,144-150 \\
18-5,144-150 \\
21-4,140-150 \\
24-5,140-150 \\
27-4,140-150 \\
30-3,144-150 \\
33-5,140-150 \\
35-5,140-150 \\
38-2,120-130\end{array}$ & $\begin{array}{r}52.0 \\
81.5 \\
129.5 \\
185.0 \\
243.5 \\
300.5 \\
357.5 \\
413.0 \\
471.5 \\
528.5 \\
585.5 \\
652.0\end{array}$ & $\begin{array}{l}7.47 \\
7.36 \\
7.06 \\
7.02 \\
7.07 \\
7.06 \\
6.96 \\
7.49 \\
7.34 \\
8.12 \\
7.91 \\
7.67\end{array}$ & $\begin{array}{r}4.53 \\
19.48 \\
18.40 \\
17.25 \\
16.69 \\
15.63 \\
12.12 \\
4.32 \\
4.52 \\
1.37 \\
1.10 \\
1.02\end{array}$ & $\begin{array}{l}(22.0) \\
19.0 \\
19.0 \\
19.2 \\
19.2 \\
19.5 \\
19.5 \\
19.4 \\
19.6 \\
19.7 \\
19.9 \\
20.4\end{array}$ & $\begin{array}{l}35.2 \\
33.3 \\
33.0 \\
33.0 \\
32.7 \\
33.6 \\
33.6 \\
33.0 \\
33.0 \\
33.0 \\
34.1 \\
34.4\end{array}$ & $\begin{array}{r}10.7 \\
5.5 \\
6.0 \\
6.9 \\
8.3 \\
9.8 \\
9.8 \\
8.6 \\
9.4 \\
9.4 \\
11.3 \\
14.6\end{array}$ & $\begin{array}{l}51.5 \\
35.7 \\
30.2 \\
28.7 \\
28.6 \\
27.2 \\
28.8 \\
26.0 \\
27.1 \\
25.8 \\
29.1 \\
27.8\end{array}$ \\
\hline \multicolumn{8}{|l|}{ Hole $362 \mathrm{~A}$} \\
\hline $\begin{array}{l}2-5,120-130 \\
3-5,120-130\end{array}$ & $\begin{array}{l}805 \\
842\end{array}$ & $7 . \overline{5}$ & $\overline{0.5}$ & $\begin{array}{l}21.0 \\
20.9\end{array}$ & $\begin{array}{l}35.5 \\
35.5\end{array}$ & $\begin{array}{l}21.0 \\
21.9\end{array}$ & $\begin{array}{l}27.5 \\
28.5\end{array}$ \\
\hline $\begin{array}{l}\text { Site } 363 \\
1-4,144-150 \\
4-1,140-150 \\
6-3,140-150 \\
9-2,140-150 \\
12-5,140-150 \\
15-1,140-150 \\
19-5,140-150 \\
23-1,140-150 \\
26-2,140-150 \\
29-5,140-150\end{array}$ & $\begin{array}{r}37.5 \\
90.0 \\
134.0 \\
189.5 \\
248.0 \\
303.5 \\
343 \\
390.5 \\
445.0 \\
504.5\end{array}$ & $\begin{array}{l}7.43 \\
7.51 \\
7.45 \\
7.30 \\
7.39 \\
7.22 \\
7.00 \\
7.31 \\
7.22 \\
-\end{array}$ & $\begin{array}{l}2.80 \\
1.78 \\
1.94 \\
1.35 \\
2.37 \\
1.60 \\
2.69 \\
0.92 \\
1.11 \\
-\end{array}$ & $\begin{array}{l}19.7 \\
19.9 \\
19.6 \\
19.8 \\
20.2 \\
20.4 \\
20.6 \\
20.2 \\
20.0 \\
20.2\end{array}$ & $\begin{array}{l}35.5 \\
35.5 \\
35.8 \\
36.0 \\
36.8 \\
36.8 \\
36.8 \\
36.3 \\
36.3 \\
36.6\end{array}$ & $\begin{array}{l}(6.4)^{\mathrm{a}} \\
13.4 \\
15.9 \\
16.8 \\
21.2 \\
23.5 \\
25.5 \\
27.1 \\
28.6 \\
34.3\end{array}$ & $\begin{array}{l}(56.4)^{\mathrm{a}} \\
49.7 \\
49.1 \\
48.2 \\
48.1 \\
46.7 \\
46.6 \\
45.1 \\
45.1 \\
43.0\end{array}$ \\
\hline \multicolumn{8}{|l|}{ Site $364^{b}$} \\
\hline $\begin{array}{l}1-3,140-150 \\
3-5,129-135 \\
5-2,125-135 \\
6-5,125-135 \\
8-5,125-135 \\
9-3,140-150 \\
12-5,140-150 \\
14-5,140-150 \\
15-3,140-150 \\
16-4,140-150 \\
\end{array}$ & $\begin{array}{r}12.5 \\
72.4 \\
156.3 \\
205.4 \\
290.1 \\
329.0 \\
376.0 \\
433.3 \\
470.0 \\
508.0 \\
\end{array}$ & $\begin{array}{l}7.48 \\
7.33 \\
7.25 \\
7.30 \\
7.25 \\
7.38 \\
7.08 \\
7.30 \\
7.39 \\
7.25 \\
\end{array}$ & $\begin{array}{l}9.03 \\
5.24 \\
3.39 \\
2.74 \mathrm{q} \\
2.24 \\
1.30 \\
1.04 \\
2.10 \\
1.31 \\
1.00\end{array}$ & $\begin{array}{l}19.4 \\
20.4 \\
21.1 \\
21.4 \\
22.8 \\
23.4 \\
25.05 \\
25.9 \\
26.0 \\
26.1\end{array}$ & $\begin{array}{l}35.2 \\
35.5 \\
38.0 \mathrm{q} \\
38.0 \\
40.2 \\
40.7 \\
42.9 \\
44.6 \\
45.7 \\
45.7\end{array}$ & $\begin{array}{r}9.5 \\
7.6 \\
15.5 \\
20.8 \\
22.2 \\
22.4 \\
25.8 \\
26.0 \\
26.1 \\
26.5 \\
\end{array}$ & $\begin{array}{l}50.9 \\
49.1 \\
50.1 \\
51.0 \\
46.3 \\
46.2 \\
44.1 \\
43.9 \\
44.5 \\
43.0\end{array}$ \\
\hline
\end{tabular}


TABLE 1 - Continued

\begin{tabular}{|c|c|c|c|c|c|c|c|}
\hline Sample & $\begin{array}{l}\text { Depth } \\
(\mathrm{m})\end{array}$ & $p \mathrm{HN}$ & $\begin{array}{l}\text { Alkalinity } \\
\text { (meq/l) }\end{array}$ & $\begin{array}{c}\text { Chlorinity } \\
(\% \circ)\end{array}$ & $\begin{array}{c}\text { Salinity } \\
(\% \circ)\end{array}$ & $\begin{array}{l}\text { Calcium } \\
(\mathrm{mM})\end{array}$ & $\begin{array}{l}\text { Magnesium } \\
\quad(\mathrm{mM})\end{array}$ \\
\hline $17-3,140-150$ & 535.0 & 6.53 & 0.90 & 26.9 & 46.8 & 26.7 & 43.1 \\
\hline $18-2,140-150$ & 557.4 & 7.16 & 1.00 & 27.9 & 47.0 & 26.4 & 43.4 \\
\hline $19-3,140-150$ & 576.0 & 7.12 & 0.65 & 27.7 & 46.8 & 26.3 & 41.4 \\
\hline $20-3,140-150$ & 584.0 & 7.63 & 0.52 & 25.7 & 44.0 & 23.8 & 41.7 \\
\hline $21-5,140-150$ & 599.5 & 7.28 & 0.51 & 28.3 & 48.4 & 27.7 & 41.4 \\
\hline $22-2,140-150$ & 622.0 & - & - & 27.2 & 46.2 & 27.9 & 40.5 \\
\hline $23-2,140-150$ & 650.5 & 7.28 & 1.07 & 29.3 & 49.5 & 27.7 & 42.2 \\
\hline $24-1,137-180$ & 680.5 & - & - & 26.9 & 45.4 & 25.5 & 40.8 \\
\hline $26-4,140-150$ & 717.0 & - & - & - & 60.5 & & \\
\hline $27-5,140-150$ & 728.0 & - & - & 35.5 & 59.4 & 37.5 & 39.6 \\
\hline $35-2,100-108$ & 899.0 & - & - & - & 71.5 & - & \\
\hline \multicolumn{8}{|l|}{ Site 365} \\
\hline $1-2,144-150$ & 228.5 & 7.39 & 15.2 & 19.9 & 35.2 & 8.9 & 50.2 \\
\hline $1, \mathrm{CC}$ & 334.5 & 7.15 & 2.34 & 30.9 & 52.8 & 23.6 & 65.2 \\
\hline $3-1,120-130$ & 402.5 & - & & 48.4 & 83.6 & 54.8 & 82.4 \\
\hline $4-1,67-76$ & 475.5 & - & & 62.0 & 102.3 & 74.1 & 81.8 \\
\hline $5-1,120-130$ & 516.3 & - & & 65.4 & 106.2 & $(74.1)$ & - \\
\hline $6-1,140-150$ & 619.5 & - & & 74.4 & 118.8 & 82.8 & 70.3 \\
\hline $7-1,128-138$ & 685.1 & - & & 75.6 & 122.1 & 82.7 & 57.4 \\
\hline
\end{tabular}

sample is representative of a very shallow level, as suggested by the Pleistocene age of the unit to which it belongs (see Chapter 4). These sediments, however, were carried from the mud line to a subbottom depth of 228 meters before the core was retrieved (see operations account in Chapter 4).

\section{CONCLUSIONS}

A major result of the interstitial water data is the consistent increase in chlorinity with depth, not only in the sites of the Angola Basin (Sites 364 and 365), but apparently also in the Southern Cape Basin (Site 361) and on the Walvis Ridge (Site 362 ). We postulate also that, for the two latter sites, salt deposits, either underlying these sediments or situated more toward the coast, are responsible for the chloride concentration increases, rather than diagenetic reactions involving water uptake in clays (c.f., Gieskes et al., 1977).

\section{REFERENCES}

Couture, R.A., Miller, R.S., and Gieskes, J.M., 1977. Interstitial water studies, Leg 41. In Seibold, E., Lancelot,
Y., et al., Initial Reports of the Deep Sea Drilling Project, Volume 41: Washington (U.S. Government Printing Office), p. 907-914.

Gieskes, J.M., 1974. Interstitial water studies, Leg 25. In Simpson, E.S.W., Schlich, R., et al., Initial Reports of the Deep Sea Drilling Project, Volume 25: Washington (U.S. Government Printing Office), p. 361-394.

Gieskes, J.M. and Lawrence, J.R., 1976. Interstitial water studies, Leg 35. In Hollister, C.D., Craddock, C., et al., Initial Reports of the Deep Sea Drilling Project, Volume 35: Washington (U.S. Government Printing Office), p. 407-424.

Gieskes, J.M., Lawrence, J.R., and Galleisky, G., 1977. Interstitial water studies, Leg 38. In Talwani, M., Udintsev, G., et al., Initial Reports of the Deep Sea Drilling Project, Volume 38: Washington (U.S. Government Printing Office).

Manheim, F.T., Waterman, L.S., Woo, C.C., and Sayles, F.L., 1974. Interstitial water studies on small core samples, Leg 23 (Red Sea). In Whitmarsh, R.B., Ross, D.A., Weser, O.E., et al., Initial Reports of the Deep Sea Drilling Project, Volume 23: Washington (U.S. Government Printing Office), p. 955-968. 

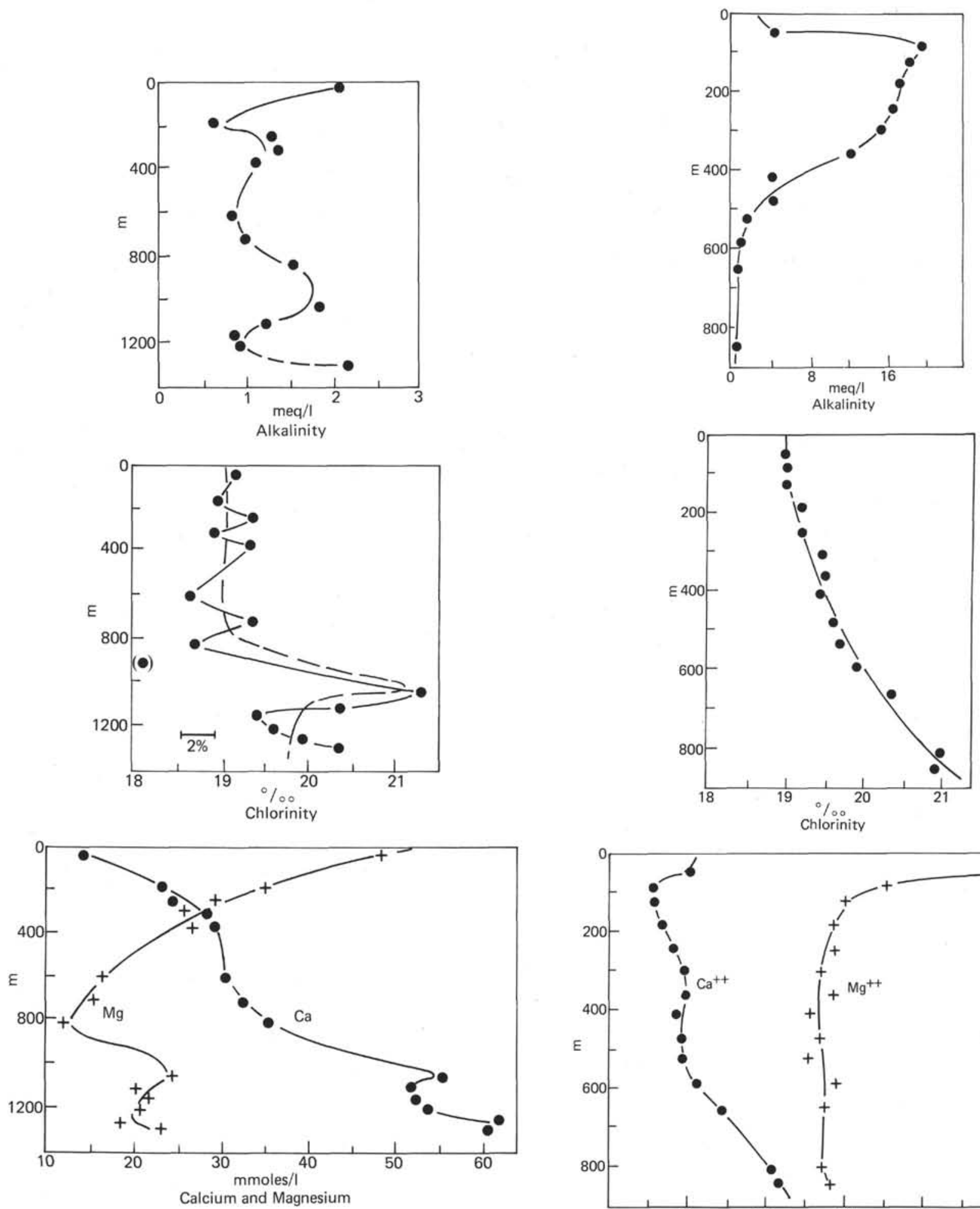

Figure 1. Interstitial water data, Site 361.

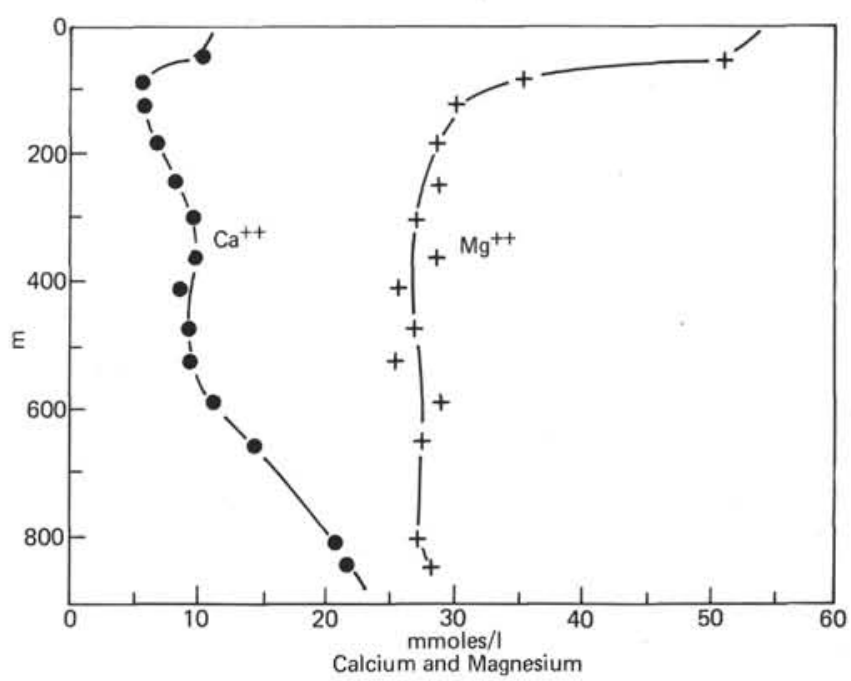

Figure 2. Interstitial water data, Site 362. 

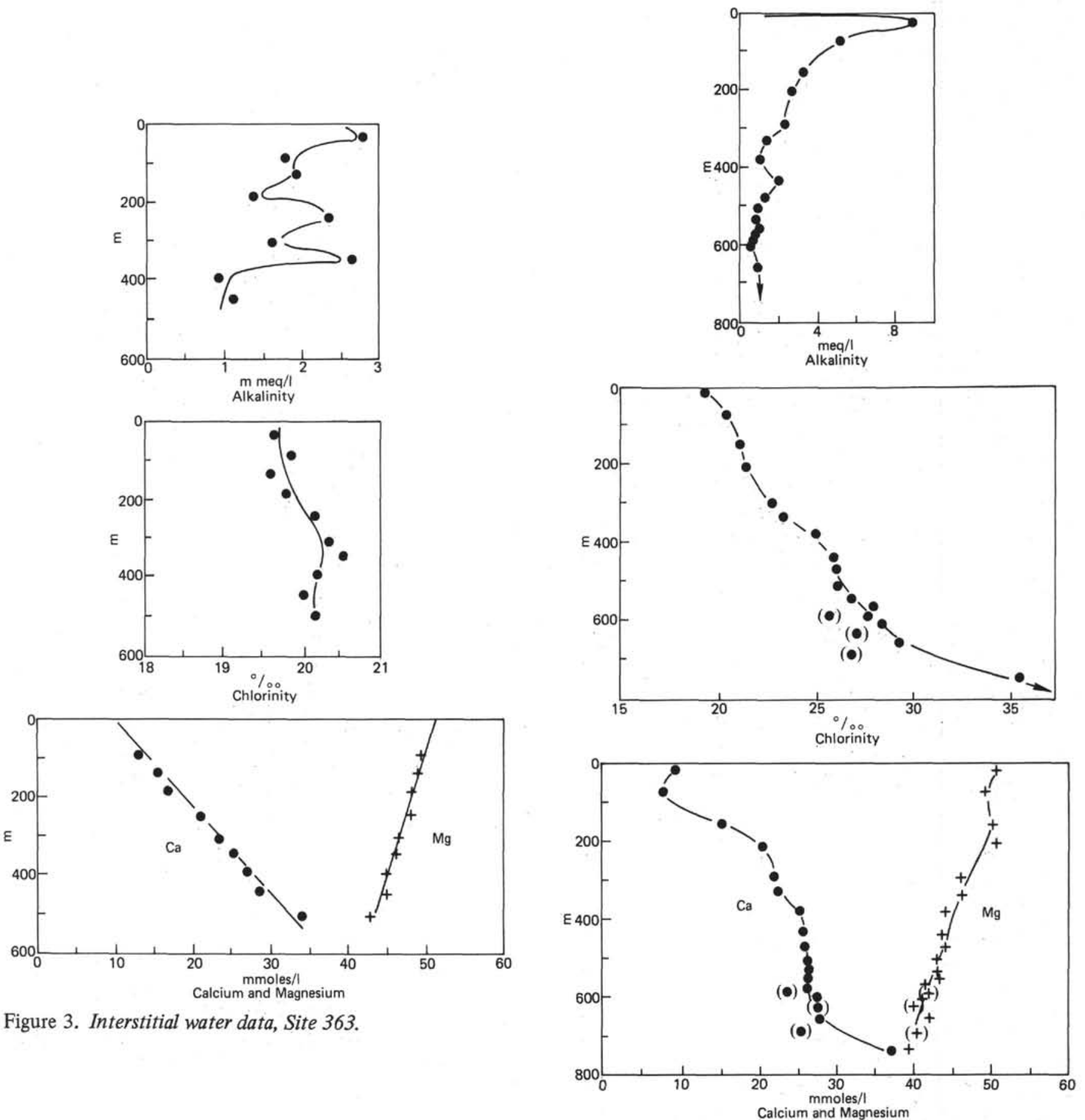

Figure 4. Interstitial water data, Site 364. 

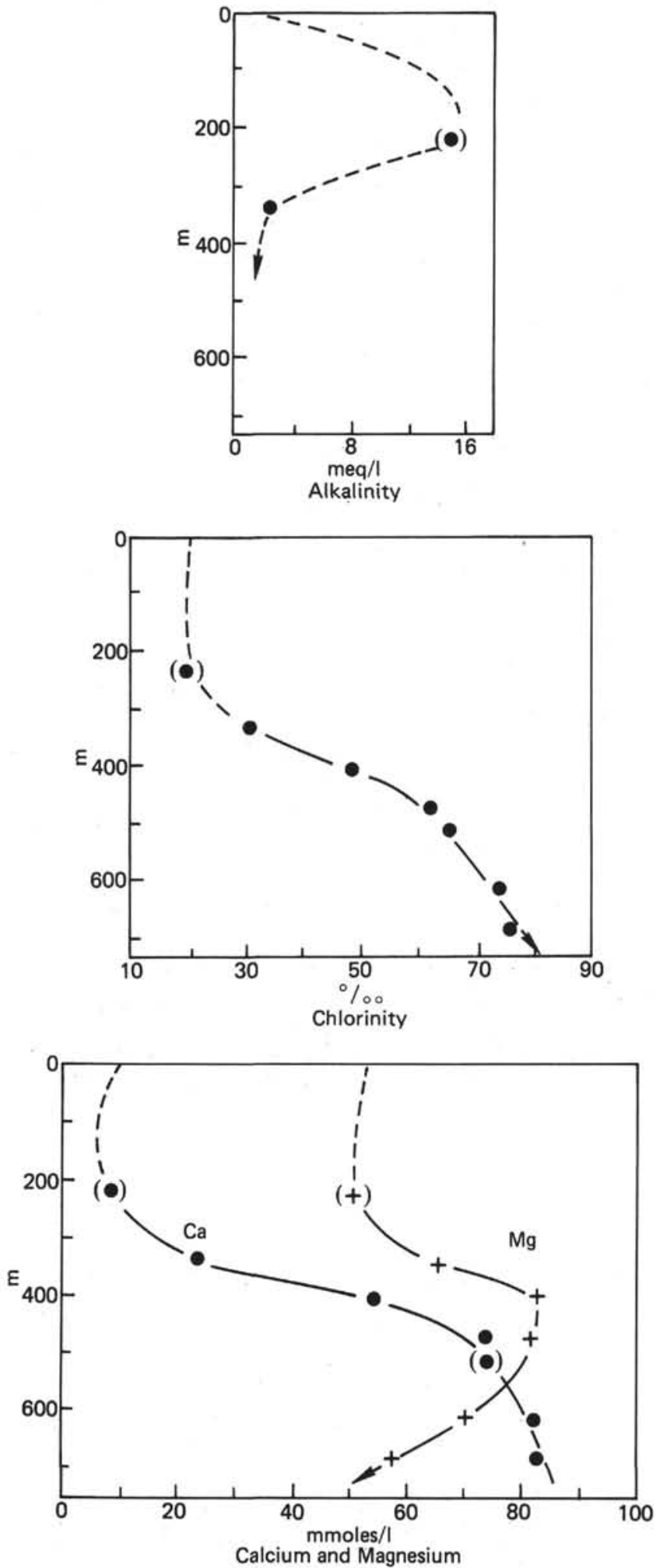

Figure 5. Interstitial water data, Site 365 . 